\title{
Quantum influence of topological defects in Gödel-type space-times
}

\author{
Josevi Carvalho ${ }^{1, \mathrm{a}}$, Alexandre M. de M. Carvalho ${ }^{2, \mathrm{~b}}$, Claudio Furtado ${ }^{3, \mathrm{c}}$ \\ ${ }^{1}$ Unidade Acadêmica de Tecnologia de Alimentos, Centro de Ciências e Tecnologia Agroalimentar, Universidade Federal de Campina Grande, \\ Pereiros, Pombal, PB 58840-000, Brazil \\ ${ }^{2}$ Instituto de Física, Universidade Federal de Alagoas, Campus A. C. Simões-Av. Lourival Melo Mota s/n, Tabuleiro do Martins, Maceió, \\ AL 57072-970, Brazil \\ ${ }^{3}$ Departamento de Física, CCEN, Universidade Federal da Paraíba, Cidade Universitária, João Pessoa, PB 58051-970, Brazil
}

Received: 20 January 2014 / Accepted: 31 May 2014 / Published online: 19 June 2014

(C) The Author(s) 2014. This article is published with open access at Springerlink.com

\begin{abstract}
In this contribution, some solutions of the KleinGordon equation in Gödel-type metrics with an embedded cosmic string are considered. The quantum dynamics of a scalar particle in three spaces whose metrics are described by different classes of Gödel solutions, with a cosmic string passing through the spaces, is found. The energy levels and eigenfunctions of the Klein-Gordon operator are obtained. We show that these eigenvalues and eigenfunctions depend on the parameter characterizing the presence of a cosmic string in the space-time. We note that the presence of topological defects breaks the degeneracy of energy levels.
\end{abstract}

\section{Introduction}

One of the most important solutions in general relativity is the Gödel metric [1], representing itself as a first cosmological solution with rotating matter. This solution is stationary, spatially homogeneous, possessing cylindrical symmetry, and its highly nontrivial property consists of breaking the causality implying the possibility of closed time-like curves (CTCs), whereas, as conjectured by Hawking [2], the presence of CTCs is physically inconsistent. Furthermore, in [35] this metric was generalized in cylindrical coordinates and the problem of causality was examined in more detail; thus it turned out that one can distinguish three different classes of solutions. These solutions are characterized by the following possibilities: (i) there are no CTCs, (ii) there is an infinite sequence of alternating causal and non-causal regions, and (iii) there is only one non-causal region. In the paper [6] the quantities called super-energy and super-momentum, which

\footnotetext{
a e-mail: josevi@ ccta.ufcg.edu.br

b e-mail: alexandre@ fis.ufal.br

c e-mail: furtado@fisica.ufpb.br
}

can be used as criteria for the possibility of the existence the CTCs, were introduced. In $[7,8]$ the CTC solutions in the Gödel space are discussed within the string context [9]. Another reason for the interest in the Gödel solution consists of the fact that the Gödel universe allows for non-trivially embedded black holes [10]. Different aspects of the Gödel solutions are discussed also in [11-14].

Recently, Drukker et al. [15] have investigated a close relation between a class of Gödel solutions for general relativity in $(3+1)$-dimensions and the Landau problem in a space of constant curvature. They solved the Klein-Gordon equation in this curved background and observed the similarity of the energy levels with the Landau problem in flat, spherical, and hyperbolic spaces. Guided by the analogy with the Landau problem, they have speculated on the possible holographic description of a single chronologically safe region. In Ref. [16] the same relation between the flat Gödel solution (Som and Raychaudhuri limit) and Landau problem in the flat space was obtained. In another way the Landau problem was investigated in several situations, for example, in a hyperbolic space $[17,18]$, in a spherical space [18], in the presence of topological defects [19-21], for neutral particles [22-25] and for non-inertial frames in a cosmic string background [26].

The cosmic string [27-30] is one of the most important examples of topological defects. In general, they can arise in a gauge theory with spontaneous symmetry breaking. They are predicted in some unified theories of particle interactions. Perhaps, they have been formed at phase transitions in the very early history of the Universe [31]. Other examples of such topological defects are the domain wall [27] and the global monopole [32]. In particular, cosmic strings provide a bridge between the physical theories for microscopic and macroscopic scales. The richness of the new ideas they 
brought about in general relativity seems to justify the interest in the study of these structures and especially the role played by their topological features for quantum systems.

In an astrophysical model, a topological defect can appear as an isolated object in empty space. Among these defects, we consider the cosmic string. In this case the conical nature of the space-time outside the defect can produce interesting physical effects. One can generalize solutions of Einstein equations in order to include the presence of a cosmic string. We use a cut-gluing method to build a solution like a cosmic string, removing an angular sector $2 \pi(1-\alpha)$. One imposes the requirement that the azimuthal angle about the axis of symmetry of the defect varies in the range $0<\phi<2 \pi \alpha$, with $\alpha=1-4 \lambda$ the disclination parameter or the deficit angle of the conical geometry, and $\lambda$ the linear mass density of the string. The parameter $\alpha$ assumes values in the interval $0<\alpha<1$ within general relativity, because in this range we deal with cosmic strings whose mass density is positive. The interval of values $\alpha>1$ is considered in geometric theory of defects in condensed matter for a negative disclination, characterized by angular excess. So, gluing the border radii of the disk with the wedge removed, we obtain the spacetime with a cosmic string passing through it. The metric of a cosmic string in polar coordinates $(t, r, \phi, z)$ is given by

$\mathrm{d} s^{2}=-\mathrm{d} t^{2}+\mathrm{d} r^{2}+\alpha^{2} r^{2} \mathrm{~d} \phi^{2}+\mathrm{d} z^{2}$.

Recently, several examples of studies in a space-time with a cosmic string were done revealing the role played by this topological defect in curved space-time. We can cite as examples, first, the Schwarzschild space-time with a cosmic string [33,34], second, the Kerr space-time with a cosmic string [35,36], third, the cosmic string in the background of AdS space [37]. In Ref. [38], a scalar quantum particle confined in two concentric thin shells in the Kerr-Newman, Gödel and Friedmann-Robertson-Walker space-times with a cosmic string passing through them has been considered in [15]. In this contribution we discuss the example of a scalar quantum particle in a class of Gödel space-times with a cosmic string passing through it. We obtain the eigenvalues and eigenfunctions of the energy. We demonstrate that the presence of a cosmic string modifies the energy levels and breaks the degeneracy of the eigenvalues.

This paper is organized as follows. In the next section we present the various solutions of the Einstein fields equations related to the values of the parameter $l^{2}$, which can assume zero, positive and negative values. In these cases, the associated geometry is flat, hyperbolic and spherical, respectively. In Sect. 3, we analyse the solutions of the Klein-Gordon equation for the Gödel space-time for three possible values assumed by $l^{2}$. We also compare the results obtained for the structures of the Landau levels (LL), discussing the degeneracy of these levels in the three cases. And in the conclud- ing remarks, we present a discussion of the main results we obtained.

\section{A Gödel-type solution}

We find that the Gödel-type space-time, in spite of its being inadequate to describe our universe, has allowed one to study many physical and mathematical models concerning gravitational backgrounds with rotation and violation of causality in general relativity; furthermore, the Gödel-type metric has the advantage that it is of rather compact form; thus, most of the calculations can be carried out analytically. A generalized discussion of the homogeneity and isotropy of the Riemannian manifolds was presented by Rebouças and Tiomno [3] with a Gödel-type metric characterized by the parameters $(l, \Omega)$. These results lead to new solutions of the Einstein fields equations, which may be called Gödel-type solutions.

Rebouças and Tiomno built a solution for a Gödel-type metric characterized by vorticity, which represents a generalization of the original Gödel metric. Since there is symmetry imposed by the cosmic strings, it is natural to use the cylindrical coordinates to describe this space-time. Thus, the general Gödel-type metrics in the presence of cosmic strings in polar coordinates $(t, r, \phi, z)$ can be written as

$$
\begin{aligned}
\mathrm{d} s^{2}= & -\left(\mathrm{d} t+\alpha \Omega \frac{\sinh ^{2} l r}{l^{2}} \mathrm{~d} \phi\right)^{2} \\
& +\alpha^{2} \frac{\sinh ^{2} 2 l r}{4 l^{2}} \mathrm{~d} \phi^{2}+\mathrm{d} r^{2}+\mathrm{d} z^{2} .
\end{aligned}
$$

The variables $(r, \phi, z, t)$ can take, respectively, the following values: $0 \leq r<\infty, 0 \leq \phi \leq 2 \pi,-\infty<(z, t)<\infty$. The parameter $\Omega$ characterizes the vorticity of the space. Note that the presence of a topological defect does not change the conditions for the existence of closed time-like curves (CTCs) in the metrics (2). This condition is the same for a Gödel-type solution in the absence of a topological defect and is given by

$\tanh \left(l r_{c}\right)=\frac{l}{\Omega}$,

where $r_{c}$ is a critical radius outside of which the CTCs can exist. The region $r<r_{c}$ is denominated as the chronologically safe one. In the expression (2) we use natural units, $c=G=1$. It is well known that in the asymptotic limit $l \rightarrow 0$ the metric has the same geometry as the SomRaychaudhuri space-time [39]. This solution of the Einstein field equations recently appeared as a Gödel-type solution in string theory. Another interesting situation is obtained when $\Omega=0$; in this case the system has zero vorticity, and the disclination parameter is $\alpha=1$. These two considerations make the metric (2) reduce to the Minkowski one. The original solution obtained by Gödel [1] can be recovered for 
$l^{2}=\Omega^{2} / 2$ and $\alpha=1$, and the anti-de Sitter case [40] corresponds to the $l^{2}=\Omega^{2}$ and $\alpha=1$ case. As pointed out by Drukker et al. [41], the physics of these kinds of metrics is closely related with the problem of a charged particle coupled to a magnetic field. The Gödel-type metrics can be written in a general form:

$\mathrm{d} s^{2}=-\left(\mathrm{d} t+A_{i}(x) \mathrm{d} x^{i}\right)^{2}+h_{i j} \mathrm{~d} x^{i} \mathrm{~d} x^{j}$,

where the spatial coordinates of the space-time are represented by the $x^{i}$. This general form represents surfaces of constant curvature in all cases $l^{2}<0, l^{2}=0$, and $l^{2}>0$ as will be shown further. The main feature of these metrics is that the geodesics in these space-times are circles which have a physical description analogous to the orbits of an electron moving in the orthogonal magnetic field known as Larmor orbits. Furthermore, the energy levels in these space-times have a Landau structure as in the Minkowski space-time with a constant curvature [15]. In the next sections we will study the influence of the cosmic string parameters on this dynamics of scalar quantum particles.

\section{The Klein-Gordon equation}

Now we will investigate a scalar quantum particle in a Gödeltype space-time. The relativistic quantum dynamics of a free spinless particle of mass $M$ is described by the Klein-Gordon equation. In its covariant form, this equation takes the following form:

$\left[\frac{1}{\sqrt{-g}} \partial_{\mu}\left(\sqrt{-g} g^{\mu \nu} \partial_{\nu}\right)-M^{2}\right] \Psi(t, \vec{r})=0$,

with $g$ being the determinant of metric tensor with inverse $g^{\mu \nu}$, and $\partial_{\mu, \nu}$ are derivatives with respect to the spatial coordinates. The $\Psi(t, \vec{r})$ is the amplitude of the probability to find the particles around the $\vec{r}$ position at the time $t$.

In this contribution, we are interested in studying solutions of the Klein-Gordon equation for a background of the Gödel-type metric with a cosmic string embedded. We will study quantum effects produced by this geometry for eigenstates and eigenfunctions. A very important parameter that must be taken into account to obtain the solutions is the sign of $l^{2}$. The case $l^{2}=0$ corresponds to a flat solution, if only $\alpha=1$, otherwise it corresponds to a spinning cosmic string. On the other hand, the case $l^{2}<0$ corresponds to a spherically symmetric solution of positive curvature, and finally the case $l^{2}>0$ corresponds to a hyperbolic solution of negative curvature. These three cases will be discussed separately in the following sections.

\subsection{Scalar particle in Som-Raychaudhuri space-time}

In this section we investigate the quantum dynamics of a scalar quantum particle in a Gödel space-time if $l^{2}=0$ in (2), also known as the Som-Raychaudhuri solution [39], so the metric (2) in this condition is reduced to

$\mathrm{d} s^{2}=-\left(\mathrm{d} t+\alpha \Omega r^{2} \mathrm{~d} \phi\right)^{2}+\alpha^{2} r^{2} \mathrm{~d} \phi^{2}+\mathrm{d} r^{2}+\mathrm{d} z^{2}$.

This solution has attracted much attention in string theory [42-44], and in [45,46] it has been interpreted as a Gödeltype solution in string theory. For this geometry, the KleinGordon equation (5) assumes the following form:

$$
\begin{gathered}
{\left[\frac{1}{r} \frac{\partial}{\partial r}\left(r \frac{\partial}{\partial r}\right)+\left(\frac{1}{\alpha r} \frac{\partial}{\partial \phi}-\Omega r \frac{\partial}{\partial t}\right)^{2}\right.} \\
\left.-\frac{\partial^{2}}{\partial t^{2}}+\frac{\partial^{2}}{\partial z^{2}}-M^{2}\right] \Psi(r)=0 .
\end{gathered}
$$

This equation is independent of time and it is symmetrical by translations along the $z$-axis, as well as by rotations, so it is reasonable to write the solution as

$$
\Psi(t, r, \phi, z)=\mathrm{e}^{-i E t+i m \phi+i k z} \Phi(r),
$$

where $E$ and $m$ are constants of separation which can be interpreted as energy and angular momentum, respectively. Substituting this ansatz into Eq. (7), we obtain the following differential equation for the radial function:

$$
\begin{gathered}
\frac{\mathrm{d}^{2} \Phi(r)}{\mathrm{d} r^{2}}+\frac{1 \mathrm{~d} \Phi(r)}{r}-\left(\frac{m^{2}}{\alpha^{2} r^{2}}+\Omega^{2} E^{2} r^{2}\right) \Phi(r) \\
+\left(E^{2}-\frac{2 m \Omega E}{\alpha}-M^{2}-k^{2}\right) \Phi(r)=0 .
\end{gathered}
$$

We can also rewrite this equation in a more appropriate way: we introduce a new variable $\xi$, defined as $\xi=\Omega E r^{2}$. Performing this change of variables we obtain

$R^{\prime \prime}(\xi)+\frac{1}{\xi} R^{\prime}(\xi)-\left(\frac{m^{2}}{4 \alpha^{2} \xi^{2}}+\frac{1}{4}-\frac{\gamma}{4 \Omega \omega \xi}\right) R(\xi)=0$.

This equation can be studied asymptotically, and its behavior at critical points, $\xi \rightarrow 0$ and $\xi \rightarrow \infty$, helps us to construct an equation in the same general form as the one for the confluent hypergeometric equation. This analysis results in

$R(\xi)=\xi^{\frac{|m|}{2 \alpha}} \mathrm{e}^{-\frac{\xi}{2}} F(\xi)$.

We obtain the following equation:

$$
\begin{aligned}
& \xi F^{\prime \prime}(\xi)+\left(\frac{|m|}{\alpha}+1-\xi\right) F^{\prime}(\xi) \\
& -\left(\frac{|m|}{2 \alpha}+\frac{1}{2}-\frac{\gamma}{4 \Omega E}\right) F(\xi)=0,
\end{aligned}
$$


with $\gamma=E^{2}-\frac{2 m \Omega E}{\alpha}-k^{2}-M^{2}$. The solution of $F(\xi)$ is a polynomial of degree $n$ obtained by the Frobenius method. Note that we require regularity and normalizability of the wave function in the origin and at infinity in Eq. (11). The exponential term in (11) implies that $\Omega E>0$ in order to ensure the condition of regularity of the wave function at infinity. In terms of the coordinate $r$ we see that (11) is written in the form $R(r) \propto r^{|m| / 2 \alpha} \mathrm{e}^{\frac{-\Omega E}{2} r^{2}} F\left(\Omega E r^{2}\right)$. Naturally, for all possible values of $\xi$ it is easy to observe that our solution is divergent at the extremal point. However, this blow-up can be eliminated by a truncation of the series. This procedure is equivalent to imposing the following condition:

$$
\left(\frac{|m|}{2 \alpha}+\frac{1}{2}-\frac{\gamma}{4 \Omega E}\right)=-n
$$

After some manipulations, we obtain the energy levels for the scalar particle in this background,

$$
\begin{aligned}
E= & \left(2 n+\frac{|m|}{\alpha}+\frac{m}{\alpha}+1\right) \Omega \\
& +\sqrt{\left(2 n+\frac{|m|}{\alpha}+\frac{m}{\alpha}+1\right)^{2} \Omega^{2}+M^{2}+k^{2}} .
\end{aligned}
$$

For $\alpha=1$, the eigenvalues are reduced to the result found by Drukker et al. [15,41]. One should notice that for $M=0$ and $k^{2}=0$ the energy levels (14) are given by

$$
E=2 \Omega\left(2 n+\frac{|m|}{\alpha}+\frac{m}{\alpha}+1\right)
$$

One should observe by Eq. (15) that the eigenvalues are similar to the ones obtained for Landau levels in the presence of a cosmic string [19]. The degeneracy of energy levels (14) is broken by the presence of the topological defect. After the normalization of the confluent hypergeometric function, we can write the expression for the eigenfunction as

$$
\begin{aligned}
\Psi(t, r, \phi, z)= & C_{n, m} \mathrm{e}^{-i E t+i m \phi+i k z} r^{\frac{|m|}{2 \alpha}} \mathrm{e}^{-\frac{\Omega E}{2} r^{2}} \\
& \times F\left(-n, \frac{|m|}{\alpha}+1, \Omega E r^{2}\right),
\end{aligned}
$$

where $C_{n, m}$ represents the normalization constant.

Due to the presence of the cosmic string, the topological parameter characterizes the eigenvalues as well as the eigenfunctions for the particle interacting with the conical geometry in Gödel-type space-time. It is evident that, in order to obtain a complete description of the interaction between scalar quantum particle and gravitational fields produced by cosmological objects, we take into account not only the local features of the background, but also the topological features.
3.2 Scalar particle in spherical symmetrical Gödel space-time

Now, we consider the limit of (2) where we can obtain a class of solutions of Gödel type possessing spherical symmetry. We suggest that $l^{2}<0$ and introduce the new coordinates in (2): $R=l / 2 l$ and $\theta=r / R$. In the case when the sign of $l^{2}$ is negative, the metric (2) takes the following form:

$$
\begin{aligned}
\mathrm{d} s^{2}= & -\left(\mathrm{d} t+\frac{\alpha \Omega r^{2}}{1+r^{2} / 4 R^{2}} \mathrm{~d} \phi\right)^{2} \\
& +\left(1+\frac{r^{2}}{4 R^{2}}\right)^{-2}\left(\mathrm{~d} r^{2}+\alpha^{2} r^{2} \mathrm{~d} \phi^{2}\right)+\mathrm{d} z^{2}
\end{aligned}
$$

Note that the second term in (17) corresponds to the metric of a two-sphere with a conical defect. In this background the Klein-Gordon equation can be written as

$$
\begin{aligned}
& {\left[\left(1+\frac{r^{2}}{4 r^{2}}\right)^{2} \frac{1}{r} \partial_{r}\left(r \partial_{r}\right)+\frac{\partial_{\phi}^{2}}{\alpha^{2} r^{2}}\right.} \\
& +\frac{1}{16 R^{4}}\left(\frac{\partial_{\phi}}{\alpha}-4 \Omega R^{2} \partial_{t}\right)^{2} r^{2}-\partial_{t}^{2}-\partial_{z}^{2}-\frac{2 \Omega \partial_{\phi} \partial_{t}}{\alpha} \\
& \left.+\frac{\partial_{\phi}^{2}}{2 \alpha^{2} R^{2}}\right] \Psi(t, \vec{r})=0 .
\end{aligned}
$$

It is easy to see that this differential equation also has a translational symmetry along the z-axis and an azimuthal symmetry. This allows us once again to write the solution as

$\Psi(t, r, \phi, z)=\mathrm{e}^{-i E t+i m \phi+i k z} \Phi(r)$.

Following this procedure, we obtain a differential equation involving only the radial variable:

$$
\begin{aligned}
& \left(1+\frac{r^{2}}{4 R^{2}}\right)^{2}\left[\frac{\mathrm{d}^{2}}{\mathrm{~d} r^{2}}+\frac{1}{r} \frac{\mathrm{d}}{\mathrm{d} r}\right] \Phi(r) \\
& -\left[\frac{m^{2}}{\alpha^{2} r^{2}}+\frac{1}{16 R^{4}}\left(\frac{m}{\alpha}+4 \Omega R^{2} E\right)^{2} r^{2}-\gamma^{\prime}\right] \Phi(r)=0,
\end{aligned}
$$

where $\gamma^{\prime}=E^{2}-\frac{2 \Omega m E}{\alpha}-k^{2}-M^{2}-\frac{m^{2}}{2 \alpha^{2} R^{2}}$. By the stereographic representation of the sphere it is possible to introduce the change of variables $r=2 R \tan \theta$. This transformation leads to the following differential equation:

$$
\begin{aligned}
& \Phi^{\prime \prime}(\theta)+\left(\frac{1}{\sin \theta \cos \theta}-\frac{2 \sin \theta}{\cos \theta}\right) \Phi^{\prime}(\theta) \\
& -\left(\frac{a^{2} \cos ^{2} \theta}{\sin ^{2} \theta}+\frac{b^{2} \sin ^{2} \theta}{\cos ^{2} \theta}-4 R^{2} \gamma^{\prime}\right) \Phi(\theta)=0,
\end{aligned}
$$

where $a=\alpha / m$ and $b=\left(m / \alpha+4 \Omega R^{2} E\right)$. By making two changes of variables, with the first one $x=\cos \theta$, and the 
second one $\xi=1-x^{2}$, we rewrite this equation as

$$
\begin{aligned}
& \xi(1-\xi) \frac{d^{2} \Phi}{d \xi^{2}}+(1-2 \xi) \frac{d \Phi}{d \xi} \\
& \quad-\left[\frac{m^{2}(1-\xi)}{4 \alpha^{2} \xi}+\frac{\lambda^{2} \xi}{4(1-\xi)}-R^{2} \gamma^{\prime}\right] \Phi=0,
\end{aligned}
$$

where $\lambda=\left|\frac{m}{\alpha}+4 \Omega R^{2} E\right|$. Let us require again that the solution must be finite at $\xi=0$ and $\xi=1$. We can write the solution as $\Phi(\xi)=(1-\xi)^{\gamma} \xi^{\beta} F(\xi)$, with the parameters $\beta=\left|\frac{m}{2 \alpha}\right|, \gamma=\frac{\lambda}{2}$. This new transformation leads to the following differential equation for the function $F(\xi)$ :

$$
\begin{aligned}
& \xi(1-\xi) \frac{\mathrm{d}^{2} F}{\mathrm{~d} \xi^{2}}+\left[\frac{|m|}{\alpha}+1-\left(\frac{2|m|}{\alpha}+4 \Omega R^{2} E+2\right) \xi\right] \\
& \times \frac{\mathrm{d} F}{\mathrm{~d} \xi}-\left[\frac{m^{2}}{\alpha^{2}}+2 \Omega R^{2}\left(\frac{|m|}{\alpha}+\frac{m}{\alpha}+1\right) E\right. \\
& \left.+\frac{|m|}{\alpha}-R^{2}\left(k^{2}+M^{2}\right)\right] F(\xi)=0 .
\end{aligned}
$$

We can identify the equations; the above equation coincides with the general form of the hypergeometric equation. Making an identification with a general hypergeometric function $F(A, B, C, \xi)$, satisfying the equation $x(1-x) F^{\prime \prime}(x)+(C-$ $(A+B+1) x) F^{\prime}(x)-A B F(x)=0$, we find the first two parameters $(A, B)$ of the hypergeometric series to be

$$
\begin{aligned}
& (A, B)=\frac{1}{2}\left(1+\frac{2|m|}{\alpha}+4 \Omega R^{2} E\right) \\
& \pm \frac{1}{2} \sqrt{1+4 \Omega R^{2} E+2 \Omega R^{2}\left(\frac{|m|}{\alpha}-\frac{m}{\alpha}\right) E+4 R^{2}\left(E^{2}-k^{2}-M^{2}\right) .}
\end{aligned}
$$

These parameters allow us to calculate the eigenvalues and eigenfunctions associated with the problem. This procedure is similar to that performed above. This enables us, after some manipulations, to find that the energy levels are given by

$$
\begin{aligned}
& E_{n, m}=\left(2 n+\frac{|m|}{\alpha}+\frac{m}{\alpha}+1\right) \Omega \\
& +\sqrt{\left(2 n+\frac{|m|}{\alpha}+\frac{m}{\alpha}+1\right)^{2} \Omega^{2}+\frac{1}{R^{2}}\left(n+\frac{|m|}{\alpha}\right)\left(n+\frac{|m|}{\alpha}+1\right)+M^{2}+k^{2}} .
\end{aligned}
$$

For each of the energy levels $E$, the set of states having the same $n$ is once again degenerate, with degenerate states being distinguished by their angular momentum $m$. However, the degeneracy of each level is now finite; there is an upper bound and a lower bound defined by the interval $-n \leq m / \alpha \leq 4 \Omega R^{2} E$. Note that the presence of topological defects reduces the degeneracy of the energy levels, since the parameter $\alpha$ has values in the range $0<\alpha<1$.

We see again that the energy levels depend on the topological parameter $\alpha$. Therefore, all information on a background space-time can be obtained by studying the eigenvalue problem. It is worth to call attention to the limit of a Minkowskian background for the spherical case studied in this section. Note that in the limit $(R \rightarrow \infty)$, the eigenvalues (25) reproduce the spectra of the particle in the Som-Raychaudhuri geometry in the presence of topological defect, and in the limit $\alpha=1$ we obtain the eigenvalues of the scalar quantum particle in the Som-Raychaudhuri background [15, 16,47].

The corresponding eigenfunction is

$$
\begin{aligned}
\Psi(t, r, \phi, z)= & C_{n, m} \mathrm{e}^{-i E t+i m \phi+i k z} r^{\frac{|m|}{2 \alpha}}\left(1-\Omega E r^{2}\right)^{\frac{1}{2}\left(\frac{|m|}{\alpha}+4 \Omega R^{2} E\right)} \\
& \times F\left(A, B, \frac{|m|}{\alpha}+1, \Omega E r^{2}\right)
\end{aligned}
$$

The constants $C_{n, m}$ are obtained from the conditions of the normalization of the wave function.

Degenerate states are present when we suppose the topological parameter to be integer. For non-integer values of $\alpha$ the degeneracy decreases in comparison with the case $\alpha=1$. Moreover, as in the Landau problem on a surface with a constant positive curvature, the spectra (25) involve a linear term accompanied by the quadratic correction introduced by the curvature, which is in accordance with the results presented in $[17,18]$ where the particles interacting with uniform magnetic field in the surface were considered.

\subsection{Hyperbolic coordinates}

Now we investigate the hyperbolic solution of (2) where $\left(l^{2}>0\right)$, which implies a hyperbolic space-time given by

$$
\begin{aligned}
\mathrm{d} s^{2}= & -\left(\mathrm{d} t+\frac{\alpha \Omega r^{2}}{1-l^{2} r^{2}} \mathrm{~d} \phi\right)^{2} \\
& +\left(1-l^{2} r^{2}\right)^{-2}\left(\mathrm{~d} r^{2}+\alpha^{2} r^{2} \mathrm{~d} \phi^{2}\right)+\mathrm{d} z^{2}
\end{aligned}
$$

In this case, the radial equation obtained from the KleinGordon equation (5) describing a scalar particle in this spacetime is written as follows:

$$
\begin{aligned}
& {\left[\left(l^{2} r^{2}-1\right)^{2}\left(\frac{\mathrm{d}^{2}}{\mathrm{~d} r^{2}}+\frac{1}{r} \frac{\mathrm{d}}{\mathrm{d} r}\right)-\frac{m^{2}}{\alpha^{2} r^{2}}\right.} \\
& \left.-l^{4}\left(\frac{m}{\alpha}-\frac{\Omega E}{l^{2}}\right)^{2} r^{2}+\gamma^{\prime}\right] \Phi(r)=0,
\end{aligned}
$$

where $\gamma^{\prime}=E^{2}+\frac{2 l^{2} m^{2}}{\alpha^{2}}-\frac{2 \Omega m E}{\alpha}-k^{2}-M^{2}$ and we have written $\Psi$ in the same form as Eq. (19). Again, one must perform a set of transformations of variables to transform the differential equation to a more suitable way. The first transformation involves the radial variable, which is now written as $r=\tanh (l \theta) / l$. It results in a new equation for the variable $\theta$ 


$$
\begin{aligned}
& \left\{\frac{\mathrm{d}^{2}}{\mathrm{~d} \theta^{2}}+\left[\frac{2 l \sinh (l \theta)}{\cosh (l \theta)}+\frac{l}{\sinh (l \theta) \cosh (l \theta)}\right] \frac{\mathrm{d}}{\mathrm{d} \theta}\right. \\
& \left.-\left[\frac{\mathcal{A}^{2} l^{2} \cosh ^{2}(l \theta)}{\sinh ^{2}(l \theta)}+\frac{l^{2} \mathcal{B}^{2} \sinh ^{2}(l \theta)}{\cosh (l \theta)}-\gamma^{\prime}\right]\right\} \Phi(\theta)=0,
\end{aligned}
$$

where $\mathcal{A}=\frac{m}{\alpha}$ and $\mathcal{B}=\frac{\Omega E}{l^{2}}-\frac{m}{\alpha}$. We also must carry out the following sequence of changes of variables: first, we introduce $y=\cosh (l \theta)$, and we denote $\xi=y^{2}-1$. With these transformations, after some algebra we arrive at

$$
\begin{aligned}
& \Phi^{\prime \prime}(\xi)+\frac{1+2 \xi}{\xi(1+\xi)} \Phi^{\prime}(\xi) \\
& -\left[\frac{\beta^{2}}{\xi^{2}}+\frac{\gamma^{2}}{(1+\xi)^{2}}-\frac{\gamma^{\prime}}{4 l^{2} \xi(1+\xi)}\right] \Phi(\xi)=0,
\end{aligned}
$$

which can be rewritten as

$$
\begin{aligned}
& \xi(1+\xi) \Phi^{\prime \prime}(\xi)+(1+2 \xi) \Phi^{\prime}(\xi) \\
& \quad-\left[\frac{\beta^{2} \xi(1+\xi)}{\xi}+\frac{\gamma^{2} \xi}{(1+\xi)}-\frac{\lambda^{2}-1}{4}\right] \Phi(\xi)=0,
\end{aligned}
$$

where $\frac{\gamma^{\prime}}{41^{2}}=\frac{\lambda^{2}-1}{4}$. The solution of this differential equation can be obtained for the ansatz

$\Phi(r)=\xi^{\beta}(1+\xi)^{\gamma} F(\xi)$,

where $\beta=|m| / 2 \alpha$ and $\gamma=\left(|m| / \alpha-\Omega E / l^{2}\right) / 2$. The solution (32) is consistent with the asymptotic conditions discussed above. Substituting (32) into (31), we obtain

$$
\begin{aligned}
& \xi(1-\xi) \frac{\mathrm{d}^{2} F}{\mathrm{~d} \xi^{2}}+\left[\frac{|m|}{\alpha}+1-\left(\frac{2|m|}{\alpha}-\frac{\Omega E}{l^{2}}+2\right) \xi\right] \frac{\mathrm{d} F}{\mathrm{~d} \xi} \\
& -\left[\frac{m^{2}}{\alpha^{2}}-\frac{\Omega}{2 l^{2}}\left(\frac{|m|}{\alpha}+\frac{m}{\alpha}+1\right)+\frac{|m|}{\alpha}-\frac{\Omega E}{2 l^{2}}\right. \\
& \left.+\frac{E^{2}-M^{2}-k^{2}}{4 l^{2}}\right] F=0 .
\end{aligned}
$$

This differential equation can easily be identified with the hypergeometric differential equation. The parameters characterizing the hypergeometric function $F(a, b, c, x)$ are given by

$$
\begin{aligned}
& (a, b)=\frac{1}{2}\left(1+\frac{2|m|}{\alpha}-\frac{\Omega E}{l^{2}}\right) \\
& \pm \frac{1}{2} \sqrt{1+\frac{\Omega^{2} E^{2}}{l^{4}}-\frac{\Omega}{2 l^{2}}\left(\frac{|m|}{\alpha}-\frac{m}{\alpha}\right) E-\frac{E^{2}-k^{2}-M^{2}}{l^{2}}} .
\end{aligned}
$$

The truncation of the series allows us to obtain the energy spectrum,

$$
\begin{aligned}
& E_{n, m}=\left(2 n+\frac{|m|}{\alpha}+\frac{m}{\alpha}+1\right) \Omega \\
& +\sqrt{\left(2 n+\frac{|m|}{\alpha}+\frac{m}{\alpha}+1\right)^{2} \Omega^{2}-4 l^{2}\left(n+\frac{|m|}{\alpha}\right)\left(n+\frac{|m|}{\alpha}+1\right)+M^{2}+k^{2}} .
\end{aligned}
$$

In the hyperbolic geometry the energy spectrum can assume discrete or continuous values $[17,47]$. The existence of these two cases is associated with the condition which parameter $\lambda$ must obey $[15,47]$. This parameter is defined by

$$
\lambda^{2}=1+\frac{\Omega^{2}-l^{2}}{l^{4}} E^{2}-\frac{\Omega}{2 l^{2}}\left(\frac{|m|}{\alpha}-\frac{m}{\alpha}\right) E+\frac{k^{2}+M^{2}}{l^{2}} .
$$

It may assume either the value $\lambda>1$ or $\lambda<1$. The condition $\lambda>1$ is satisfied when $\Omega^{2}>l^{2}$, and, as discussed by [47], the corresponding spectra belong to a region with discrete energy levels. For $\Omega^{2}<l^{2}$, the discrete energy levels are bounded from above by the energy

$$
\begin{aligned}
E \leq & \frac{\Omega l^{2}}{l^{2}-\Omega^{2}}\left(\frac{|m|}{\alpha}-\frac{m}{\alpha}\right) \\
& \pm \sqrt{\frac{\Omega^{2} l^{4}}{\left(l^{2}-\Omega^{2}\right)^{2}}\left(\frac{|m|}{\alpha}-\frac{m}{\alpha}\right)^{2}+\frac{l^{2}\left(k^{2}+m^{2}\right)}{l^{2}-\Omega^{2}}} .
\end{aligned}
$$

Note that the condition (37) is satisfied for the upper sign case by a positive energy and for the case of the lower sign is satisfied by a negative energy. Above this limit, the spectrum is continuous.

The eigenstates corresponding to the discrete spectra can be written in the form

$$
\begin{aligned}
\Psi(t, r, \phi, z)= & C_{n, m} \mathrm{e}^{-i E t+i m \phi+i k z} r^{\frac{|m|}{2 \alpha}}\left(1+\Omega E r^{2}\right)^{\frac{1}{2}\left(\frac{|m|}{2 \alpha}-\frac{\Omega E}{l^{2}}\right)} \\
& \times F\left(a, b, \frac{|m|}{\alpha}+1, \Omega E r^{2}\right),
\end{aligned}
$$

for $0 \leq m / \alpha \leq \infty$, and

$$
\begin{aligned}
\Psi(t, r, \phi, z)= & C_{n, m} \mathrm{e}^{-i E t+i m \phi+i k z} r^{-\frac{|m|}{2 \alpha}}\left(1+\Omega E r^{2}\right)^{-\frac{1}{2}\left(\frac{|m|}{2 \alpha}-\frac{\Omega E}{l^{2}}\right)} \\
& \times F\left(a, b, \frac{|m|}{\alpha}+1, \Omega E r^{2}\right),
\end{aligned}
$$

for $-n \leq m / \alpha \leq 0$.

Otherwise, we have continuous spectra, where the condition for $n$ following from the restriction $\lambda<1$ is given by the expression

$$
\left(2 n+\frac{|m|}{\alpha}+\frac{m}{\alpha}+1\right) \leq \frac{\Omega}{l} \sqrt{\frac{M^{2}+k^{2}}{l^{2}-\Omega^{2}}-\frac{\Omega}{2}\left(\frac{|m|}{\alpha}-\frac{m}{\alpha}\right)},
$$

and above this limit the continuous solutions appear with the energy spectra obeying Eq. (35). 
In order to summarize this section, we call attention to the fact that we obtained two distinct behaviors of the energy spectra. One case corresponds to the region described by $\Omega^{2}>l^{2}$, which the closed time-like curves can present in a classical background. The spectrum of the energy associated with this region is discrete. The other observed behavior corresponds to $\Omega^{2}<l^{2}$. The corresponding space-time is free of closed time-like curves. In this case we have a discrete energy level upper bound by a continuous spectrum obeying Eq. (37) and the quantum number $n$ is restricted by the condition (40).

\section{Conclusion}

We studied the impact of the presence of topological defects in the family of Gödel-type space-times on the energy levels of a scalar quantum particle in this geometry. We have solved the Klein-Gordon equation in the spherical, hyperbolic and flat geometries and found that the eigenvalues and eigenfunctions depend of the parameter characterizing the presence of a cosmic string in the Gödel-type background space-time. The presence of the parameter $\alpha$ breaks the degeneracy of energy levels in the three cases: Som-Raychaudhuri, spherical Gödel and hyperbolic Gödel solutions. The presence of topological defects in the Gödel-type space-time has an impact on the levels of the energy for the scalar particle. The presence of defects leads to a separation between the energy levels. This would be a theoretical prediction to detect the presence of a cosmic string by a study of the break of the degeneracy of the energy spectrum of the particle in this type of background. We have to keep in mind that this is an idealized model, and a more realistic model would investigate the energy levels of atoms or molecules in these space-times.

Comparing our results with the conclusions of the paper [15], we find that at the classical level all solutions, corresponding to a discrete energy with $n$ bounded from above by (40) for $\Omega^{2}>l^{2}$ and unbounded in the opposite case, coincide with the results for the range of parameters $(\Omega, l)$ in which the closed time-like curves can exist for a classical geodesic's motion in the hyperbolic geometry, while the movement in regions free of CTCs with unbounded orbits is equivalent, at the quantum level, to regions with continuous energy states. Even for this relation for the classical and quantum levels we have another problem, which has a physical content analogous to the Gödel-type solution on hyperbolic geometries, that is, the Landau problem on surfaces of constant curvature. In this case, the discrete energy levels are obtained for a strong enough magnetic field, which forces the electron to follow a closed orbit. The unbounded orbits run by the particle are associated with the continuous energy spectra, and the classical motion is free of closed time-like curves as discussed earlier. We finalize this contri- bution calling to the reader's attention that the results with Gödel-type metrics found in $[39,41]$ can be obtained in the limits $\left(R \rightarrow \infty, l^{2} \rightarrow 0\right)$ and $\alpha=1$.

Acknowledgments We thank CNPQ, CAPES, FAPESQ, CAPES, for the financial support.

Open Access This article is distributed under the terms of the Creative Commons Attribution License which permits any use, distribution, and reproduction in any medium, provided the original author(s) and the source are credited.

Funded by $\mathrm{SCOAP}^{3}$ / License Version CC BY 4.0.

\section{References}

1. K. Gödel, Rev. Mod. Phys. 21, 447 (1949)

2. S. Hawking, Phys. Rev. D 46, 603 (1992)

3. M. Rebouças, J. Tiomno, Phys. Rev. D 28, 1251 (1983)

4. M. Rebouças, M. Aman, A.F.F. Teixeira, J. Math. Phys. 27, 1370 (1985)

5. M.O. Galvao, M. Rebouças, A.F.F. Teixeira, W.M. Silva Jr, J. Math. Phys. 29, 1127 (1988)

6. M. Dabrowski, J. Garecki, Class. Quantum Gravity 19, 1 (2002). gr-qc/0102092

7. J. Barrow, M. Dabrowski, Phys. Rev. D 58, 103502 (1998). gr-qc/9803048

8. P. Kanti, C.E. Vayonakis, Phys. Rev. D 60, 103519 (1999). gr-qc/9905032

9. O. Bertolami, F. Lobo, Neuro. Quantol. 7, 1 (2009). arXiv: 0902.0559 [gr-qc]

10. X. He, B. Wang, S. Chen, Phys. Rev. D 79, 084005 (2009). arXiv: 0811.2322 [gr-qc]

11. J.D. Barrow, C. Tsagas, Class. Quantum Gravity 21, 1773 (2004). gr-qc/0308067

12. J.D. Barrow, C. Tsagas, Phys. Rev. D 69, 064007 (2004). gr-qc/0309030

13. T. Clifton, J. Barrow, Phys. Rev. D 72, 123003 (2005). gr-qc/0511076

14. R.J. Gleiser, M. Gurses, A. Karasu, S. Özgür, Class. Quantum Gravity 23, 2653 (2006)

15. N. Drukker, B. Fiol, J. Simón, JCAP 0410, 012 (2004)

16. S. Das, J. Gegenberg, Gen. Relativ. Gravit. 40, 2115 (2008)

17. A. Comtet, Ann. Phys. 173, 185 (1987)

18. G.V. Dunne, Ann. Phys. 215, 233 (1992)

19. C. Furtado, B.C.G. da Cunha, F. Moraes, E.R. Bezerra de Mello, V.B. Bezerra, Phys. Lett. A 21, 90 (1994)

20. C. Furtado, F. Moraes, Europhys. Lett. 45, 279 (1999)

21. A.L. Silva Netto, C. Furtado, J. Phys Condens. Matter 20, 125209 (2008)

22. M. Ericsson, E. Sjöqvist, Phys. Rev. A 65, 013607 (2001)

23. L.R. Ribeiro, C. Furtado, J.R. Nascimento, Phys. Lett. A 348, 135 (2006)

24. L.R. Ribeiro, C. Furtado, J.R. Nascimento, Phys. Lett. A 358, 336 (2006)

25. K. Bakke, C. Furtado, Phys. Rev. A 80, 032106 (2009)

26. K. Bakke, C. Furtado, Phys. Rev. D 82, 084025 (2010)

27. A. Vilenkin, Phys. Rep. 121, 263 (1985)

28. A. Vilenkin, Phys. Lett. B 133, 177 (1983)

29. W.A. Hiscock, Phys. Rev. A 31, 3288 (1985)

30. B. Linet, Gen. Relativ. Gravit. 17, 1109 (1985)

31. T.W.B. Kibble, J. Phys. A 19, 1387 (1976)

32. M. Barriola, A. Vilenkin, Phys. Rev. Lett. 63, 341 (1989)

33. M. Aryal, L.H. Ford, A. Vilenkin, Phys. Rev. D 34, 2263 (1986) 
34. M.G. Germano, V.B. Bezerra, E.R. Bezerra de Mello, Class. Quantum Gravity 13, 2663 (1996)

35. D.V. Gal'tsov, E. Masar, Class. Quantum Gravity 6, 1313 (1989)

36. N. Özdemir, Class. Quantum Gravity 20, 4409 (2003)

37. E.R. Bezerra de Mello, A.A. Saharian, J. Phys. A Math. Theor. 45, $115402(2012)$

38. S.G. Fernandes, G. de A Marques, V.B. Bezerra, Class. Quantum Gravity 23, 7063 (2006)

39. M.M. Som, A.K. Raychaudhuri, Proc. R. Soc. A 304, 81 (1968)

40. M. Rooman, P. Spindel, Class. Quantum Gravity 15, 3241 (1998). gr-qc/9804027
41. N. Drukker, B. Fiol, J. Simón, Phys. Rev. Lett. 91, 231601 (2003)

42. G.T. Horowitz, A.A. Tseytlin, Phys. Rev. D 51, 2896 (1995)

43. G. Russo, A.A. Tseytlin, Nucl. Phys. B 448, 293 (1995)

44. J.G. Russo, A.A. Tseytlin, Nucl. Phys. B 454, 164 (1995)

45. E.K. Boyda, S. Ganguli, P. Horava, U. Varadarajan, Phys. Rev. D 67, $106003(2003)$

46. T. Harmark, T. Takayanagi, Nucl. Phys. B 662, 3 (2003)

47. B.D. Figueiredo, I.D. Soares, J. Tiomno, Class. Quantum Gravity 9, 1593 (1992) 